\title{
Publisher Correction: Specification curve analysis
}

Uri Simonsohn (1D, Joseph P. Simmons and Leif D. Nelson (1)

Correction to: Nature Human Behaviour https://doi.org/10.1038/s41562-020-0912-Z, published online 27 July 2020.

In the version of this article initially published, significant discrepancies appeared between the HTML and PDF versions of the article. The HTML version was (and remains) correct, but equations in the PDF were missing bidirectional arrows over many elements. The errors have been corrected in the PDF version of the article.

Published online: 9 October 2020

https://doi.org/10.1038/s41562-020-00974-w

(c) The Author(s), under exclusive licence to Springer Nature Limited 2020 